\title{
Predictive relevance of PD-L1 expression with pre-existing Taper in gastric cancer
}

\author{
Xiaoli Juํ, Rong Shen ${ }^{1,2}$, Pan Huang ${ }^{1}$, Jianhua Zhai ${ }^{1}$, Xiaobin Qian ${ }^{1}$, Qiang Wang ${ }^{3}$ \\ and Miao Chen ${ }^{1,2}$ \\ ${ }^{1}$ Department of Pathology, School of Medicine, Jiangsu University, Zhenjiang, Jiangsu 212013, P.R. China \\ ${ }^{2}$ Department of Pathology, The Affiliated People's Hospital of Jiangsu University, Zhenjiang, Jiangsu 212013, P.R. China \\ ${ }^{3}$ Institute of Life Sciences, Jiangsu University, Zhenjiang, Jiangsu 212013, P.R. China \\ Correspondence to: Qiang Wang, email: wangqiang@ujs.edu.cn \\ Miao Chen, email: 18912802271@163.com
}

Keywords: PD-L1; gastric cancer; TILs; prognosis

Received: January 12, $2017 \quad$ Accepted: October 11, $2017 \quad$ Published: October 26, 2017

Copyright: Ju et al. This is an open-access article distributed under the terms of the Creative Commons Attribution License 3.0 (CC BY 3.0), which permits unrestricted use, distribution, and reproduction in any medium, provided the original author and source are credited.

\section{ABSTRACT}

Expression of programmed cell death receptor ligand 1 (PD-L1) has been shown to be up-regulated in some gastric cancer patients and to correlate with the density of tumour infiltrating lymphocytes (TILs). However, conflicting results have been reported regarding TILs and the expression of PD-L1 as a prognostic marker for gastric cancer. We investigated the correlation of PD-L1 and TILs expression with clinicpathological characteristics in $\mathbf{1 0 5}$ well characterized gastric cancer patients. PD-L1 expression and CD3+ and CD8+ TILs were evaluated by fluorescent multiplex immunohistochemistry ( $\mathrm{mIHC}$ ) analysis. PD-L1 positive staining on tumour cells was observed in $35 \%$ cases and $48 \%$ cases showed PD-L1 expression on immune cells. Up-regulated PD-L1 expression on tumour cells and immune cells was associated with high density of pre-existing tumour infiltrating $\mathrm{CD} 3+$ and $\mathrm{CD} 8+$. In additional, more than $70 \%$ tumor infiltrating CD3+ cells were CD3+CD8+ cells. More than $60 \%$ PD-L1+ immune cells were PD-L1+CD3+CD8+ cells. PD-L1 expression in tumour cells was associated with poor prognosis and high density CD3+ and CD8+ TILs indicated improved overall survival in gastric cancer patients. Increased PD-L1 expression with low density CD3+ and CD8+ TILs had the shortest overall survival. In accordingly, PD-L1 absence with high density CD3+ and CD8+ TILs indicated the best prognosis. Combination of PD-L1 with pre-existing TILs may be more precise than PD-L1 alone for predicting survival in gastric cancer.

\section{INTRODUCTION}

Gastric cancer (GC) is the fourth most common cancer and second leading cause of cancer associated death for men and women worldwide [1,2]. Although multidisciplinary therapeutic strategies have improved treatment outcomes, the overall prognosis for gastric cancer patients remains poor. Currently, blocking the programmed death 1 (PD-1) and it's ligand 1 (PD-L1) immune checkpoint signalling to restore anti-tumour immunity has shown unprecedented rates of durable clinical responses in patients, notably in melanoma, renal, lung, prostate and bladder carcinomas [3-6]. Phase Ib studies of immunotherapy for advanced gastric cancer (KEYNOTE-012) are ongoing, with $22 \%$ of patients recorded having an overall response by blocking the PD-1/ PD-L1 immune checkpoint [7].

PD-1 is present on the surface of active T and B cells, and PD-L1 is expressed on many types of immune cells. Several tumour types express PD-L1, including gastric cancer $[8,9]$. The Interaction of PD- 1 and PD$\mathrm{L} 1$ suppresses the $\mathrm{CD} 8+\mathrm{T}$ cells immune response, 
induces an immunosuppressive microenvironment within the tumour, and allows the tumour to evade immune destruction [10, 11]. Using an IHC approach, PD-L1 expression was detected in $12 \%-40 \%$ of gastric cancer samples, was only weakly detectible in gastric adenomas, and undetectable in normal gastric tissue controls $[12,13]$. Several studies have reported a correlation between PD-L1 expression and the prognosis of cancer patients, and that PD-L1 expression is a predictive biomarker for blocking PD-1/PD-L1 treatment response [13-15]. However, the clinical implications of the existence of PD-L1 in tumours and TILs in the tumour microenvironment are still controversial, and the prognostic potential of these factors is unclear.

There are several reports suggesting that PD-L1 expression is associated with poor prognosis of gastric patients [16-18]. Gastric cancer patients with higher intratumoural and stromal CD8+ T cell density also have higher PD-L1 expression, which is associated with shorter progression free and overall survival times [16]. Patients with enhanced expression of FoxP3 and PD-L1 exhibited a lower overall survival rate and a worse prognosis [18]. There are also many reports demonstrating that PD-L1 expression is associated with good prognosis of gastric cancer patients. In gastric cancer cases of western patients, high PD-L1/PD-1 expression was associated with a significantly better patient outcome [13]. High PD-L1 expression and high density of $\mathrm{CD} 3+\mathrm{T}$ cells in the tumour microenvironment are better prognostic markers in GC 17. GC patients with high density CD8+ and FoxP3+ TILs showed significantly higher overall survival rates than GC patients with low density CD8+ or FoxP3+ cells [19]. With these conflicting results, the true relationship of PD-L1 expression and TILs with patient clinical outcome is yet to be clarified.

We investigated the expression of PD-L1, CD8 and CD3 in gastric tumour specimens and evaluated the relationship of these factors to clinicopathological characteristics and patient survival. These results may serve as a surrogate marker for PD-L1-positive GCs and may help in validating biomarkers to select patients for immune checkpoint treatment strategies.

\section{RESULTS}

\section{PD-L1 expression in GC (IHC)}

We examined PD-L1 expression levels in tumour and immune cells in 105 gastric cancer specimens, but not in non-neoplastic gastric epithelium. We observed that PDL1 was mainly expressed on the membrane and cytoplasm of tumour and immune cells (Figure 1A, 1F). In the present

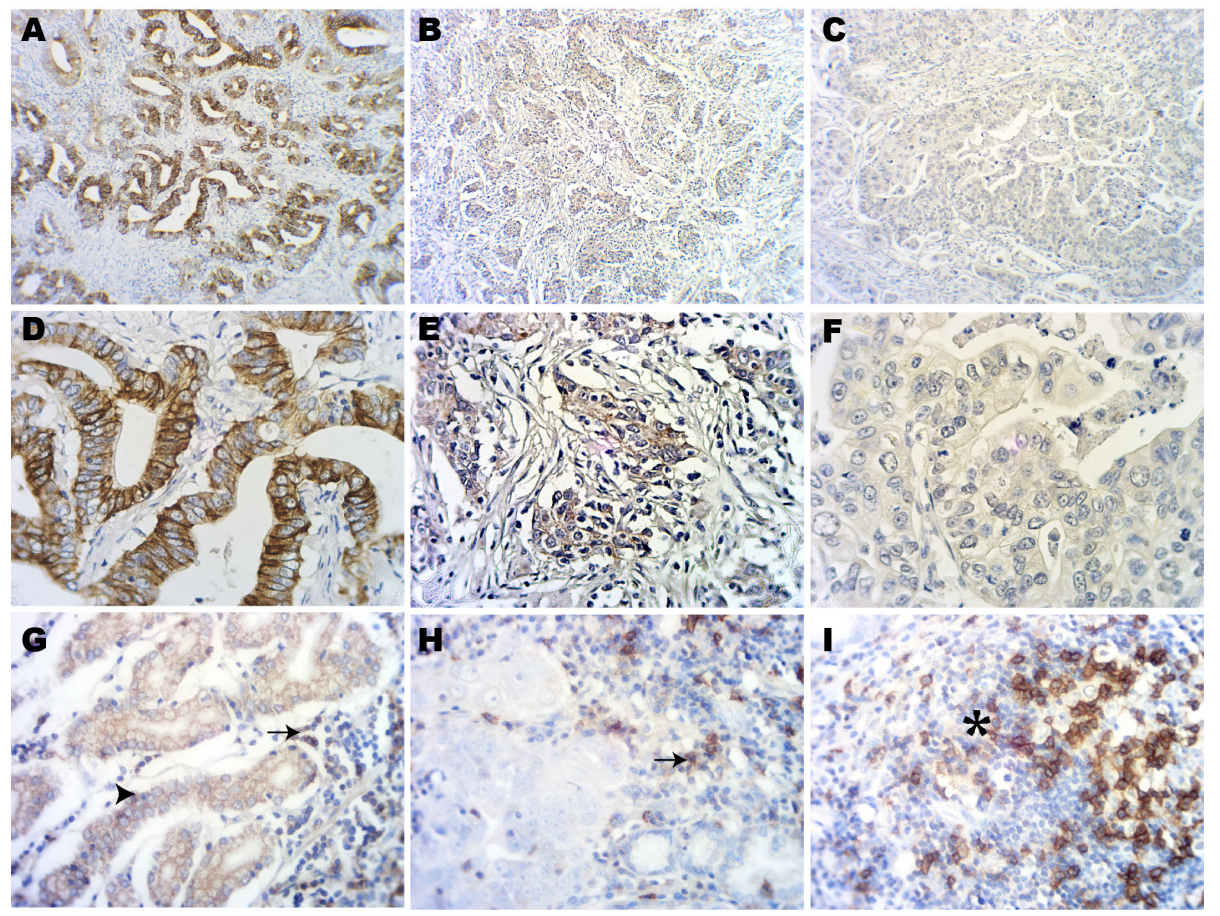

Figure 1: Immunohistochemical staining of PD-L1 in gastric adenocarcinoma tissues. (A) Strong expression of PDL1 on tumour cells. (B) Weak expression of PD-L1 on tumour cells. (C) Negative expression of PD-L1 on tumour cells. (D) Strong expression of PD-L1 on tumour cells. (E) Weak expression of PD-L1 on tumour cells. (F) Negative expression of PD-L1on tumour cells. (G) Expression of PD-L1 in tumour cells (arrowhead) and intratumoural immune cells (arrow). (H) Expression of PD-L1 on intratumoural immune cells (arrowhead) but not on tumour cells. (I) Expression of PD-L1 on intratumoural lymph follicles (star). (A-C original magnification $\times 100$. D-I original magnification $\times 400)$. 
Table 1: Clinical, pathological characteristics and their correlation with PD-L1 expression

\begin{tabular}{|c|c|c|c|c|c|c|c|}
\hline \multirow[t]{2}{*}{ Characteristic } & \multirow{2}{*}{$\begin{array}{l}\text { Total no } \\
(n=105)\end{array}$} & \multicolumn{2}{|c|}{$\begin{array}{l}\text { PD-L1 in tumor } \\
\text { cells }\end{array}$} & \multirow[t]{2}{*}{$P$-Value } & \multicolumn{2}{|c|}{$\begin{array}{l}\text { PD-L1 in immune } \\
\text { cells }\end{array}$} & \multirow[t]{2}{*}{$P$-Value } \\
\hline & & Negative & Positive & & Negative & Positive & \\
\hline $\begin{array}{l}\text { Mean age } \pm \text { SD (year) } \\
\text { Total no }\end{array}$ & $\begin{array}{l}63.9 \pm 9.6 \\
105(100)\end{array}$ & $\begin{array}{l}64.1 \pm 8.5 \\
68(65 \%)\end{array}$ & $\begin{array}{c}63.2 \pm 14.0 \\
37(35 \%)\end{array}$ & 0.970 & $\begin{array}{c}63.8 \pm 13.1 \\
55(52 \%)\end{array}$ & $\begin{array}{l}64.2 \pm 8.1 \\
50(48 \%)\end{array}$ & 0.925 \\
\hline Gender & & & & 0.082 & & & 0.051 \\
\hline Female & $21(20 \%)$ & $17(81 \%)$ & $4(19 \%)$ & & $15(71 \%)$ & $6(29 \%)$ & \\
\hline Male & $84(80 \%)$ & $51(61 \%)$ & $33(39 \%)$ & & $40(48 \%)$ & $44(52 \%)$ & \\
\hline Location & & & & 0.012 & & & 0.007 \\
\hline Proximal & $30(29 \%)$ & $25(83 \%)$ & $5(17 \%)$ & & $22(73 \%)$ & $8(27 \%)$ & \\
\hline Antral & $75(71 \%)$ & $43(57 \%)$ & $32(43 \%)$ & & $33(44 \%)$ & $42(56 \%)$ & \\
\hline AJCC stage & & & & 0.997 & & & 0.453 \\
\hline I & $29(28 \%)$ & $19(66 \%)$ & $10(34 \%)$ & & $18(62 \%)$ & $11(38 \%)$ & \\
\hline II & $33(31 \%)$ & $21(64 \%)$ & $12(36 \%)$ & & $17(52 \%)$ & $16(48 \%)$ & \\
\hline III & $31(30 \%)$ & $20(65 \%)$ & $11(35 \%)$ & & $13(42 \%)$ & $18(58 \%)$ & \\
\hline IV & $12(11 \%)$ & $8(67 \%)$ & $4(33 \%)$ & & $7(58 \%)$ & $5(42 \%)$ & \\
\hline Tumor differentiation & & & & 0.016 & & & 1.0 \\
\hline Poor & $63(60 \%)$ & $35(56 \%)$ & $28(44 \%)$ & & $33(52 \%)$ & $30(48 \%)$ & \\
\hline Moderate to well & $42(40 \%)$ & $33(79 \%)$ & $9(21 \%)$ & & $23(52 \%)$ & $20(48 \%)$ & \\
\hline $\begin{array}{l}\text { Tumor growth } \\
\text { pattern }\end{array}$ & & & & 0.183 & & & 0.123 \\
\hline Expansile & $3(3 \%)$ & $3(100 \%)$ & $0(0 \%)$ & & $3(100 \%)$ & $0(0 \%)$ & \\
\hline Intermediate & $72(69 \%)$ & $43(60 \%)$ & $29(40 \%)$ & & $34(47 \%)$ & $38(53 \%)$ & \\
\hline Infiltrative & $30(29 \%)$ & $22(73 \%)$ & $8(27 \%)$ & & $18(60 \%)$ & $12(40 \%)$ & \\
\hline Ki67 status & & & & 0.022 & & & 0.004 \\
\hline low & $47(46 \%)$ & $36(77 \%)$ & $11(23 \%)$ & & $32(68 \%)$ & $15(32 \%)$ & \\
\hline high & $58(54 \%)$ & $32(55 \%)$ & $26(45 \%)$ & & $23(40 \%)$ & $35(60 \%)$ & \\
\hline HER-2 status & & & & 0.022 & & & 0.134 \\
\hline Negative & $86(82 \%)$ & $60(70 \%)$ & $26(30 \%)$ & & $48(56 \%)$ & $38(44 \%)$ & \\
\hline Positive & $19(18 \%)$ & $8(42 \%)$ & $11(58 \%)$ & & $7(37 \%)$ & $12(63 \%)$ & \\
\hline
\end{tabular}

study, 37 of 105 cases (35\%) exhibited PD-L1 positive staining (Table 1). Although $90 \%$ of the cases showed the same staining intensity within the same sample, different staining intensities were observed between different samples (Figure 1A, 1B, 1D, 1E). Samples with $<5 \%$ PDL1 stained tumour cells were considered PD-L1 negative (Figure 1C, 1F) as previous reports [16]. After examining all of the samples, we observed that of PD-L1 positive tumour cell samples, PD-L1 staining in 5\% to $65 \%$ of tumor cells.

We also evaluated PD-L1 expression on immune cells. PD-L1 staining in $\geq 1 \%$ of immune cells was considered PD-L1 positive. Fifty of 105 cases (48\%) were PD-L1 expression (Table 1). There were 35 cases were PD-L1 positive both on tumour infiltrating immune cells and tumour cells (Figure 1G) and 15 cases PD-
L1 expressed on immune cells but not on tumour cells (Figure 1H). PD-L1 positive immune cells were present on intratumoral lymph follicles immune cells (Figure 1I). Of the PD-L1 positive sample, PD-L1 staining was present in $1 \%$ to $50 \%$ of the immune cells.

\section{Correlation between PD-L1 expression and clinicopathological features}

Clinicopathological features and molecular characteristics according to PD-L1 expression on tumour cells and immune cells are summarized in Table 1.

The study cohort $(n=105)$ included 84 males $(80 \%)$ and 21 females $(20 \%)$. According to age, patients were classified into $<65 y(49 \%)$ and $\geq 65$ y (51\%) subsets. Expression of PD-L1 in tumour cells was associated with 
location $(P=0.012)$, tumour differentiation $(P=0.016)$, Ki67 status $(P=0.022)$ and HER-2 status $(P=0.022)$. In 30 cases $(29 \%)$, tumours were located in the gastric cardia and body (17\% had PD-L1 positive tumour cells). In 75 cases $(71 \%)$, tumours were located in the gastric antrum (43\% had PD-L1 positive tumour cells). Fortytwo cases were moderate to well differentiated $(21 \%$ had PD-L1 positive tumour cells), and 63 cases were poor differentiated (44\% had PD-L1 positive tumour cells). Expression of PD-L1 in tumour cells was significantly associated with the high Ki67 and HER-2 positive cases. In Ki67 high status cases approximately 45\% had PDL1 positive tumour cells. However, in Ki67 low subsets $23 \%$ had PD-L1expression in tumour cells. In HER-2 positive cases approximately $58 \%$ had PD-L1 positive tumour cells. However, in HER-2 negative subsets less than $30 \%$ had PD-L1 positive tumour. Expression of PD-L1 in immune cells was also associated with antral location $(P=0.007)$ and high Ki67 subtype $(P=0.004)$. Interestingly, in Ki67 high cases approximately $60 \%$ had PD-L1 positive immune cells. However, in Ki67 low cases $32 \%$ had PD-L1 positive immune cells. Unlike in the tumour cells, expression of PD-L1 in immune cells was not significantly associated with the poor differentiation or HER-2 status.

Staging was classified according to the tumournode-metastasis (TNM) classification of the American Joint Committee on Cancer (AJCC, 7th edition). PD-L1 expression was not significantly associated with age, gender, disease stage or tumour growth pattern on either tumour cells or on immune cells.

\section{Correlation between PD-L1 and TILs (mIHC)}

Increased PD-L1 expression on tumor cells and immune cells both positively correlated with CD3+ and CD8+ cell infiltration in gastric cancer. PD-L1 positive in tumour cell subset had a high density of tumor infiltrating CD3+ cells and CD8+ cells (Figure 2A). And more than $70 \% \mathrm{CD} 3+$ cells were CD3 + CD8+ cells (Figure 2B). PD-L1 negative in tumour cell subset had a low density of tumor infiltrating CD3+ cells and CD8+

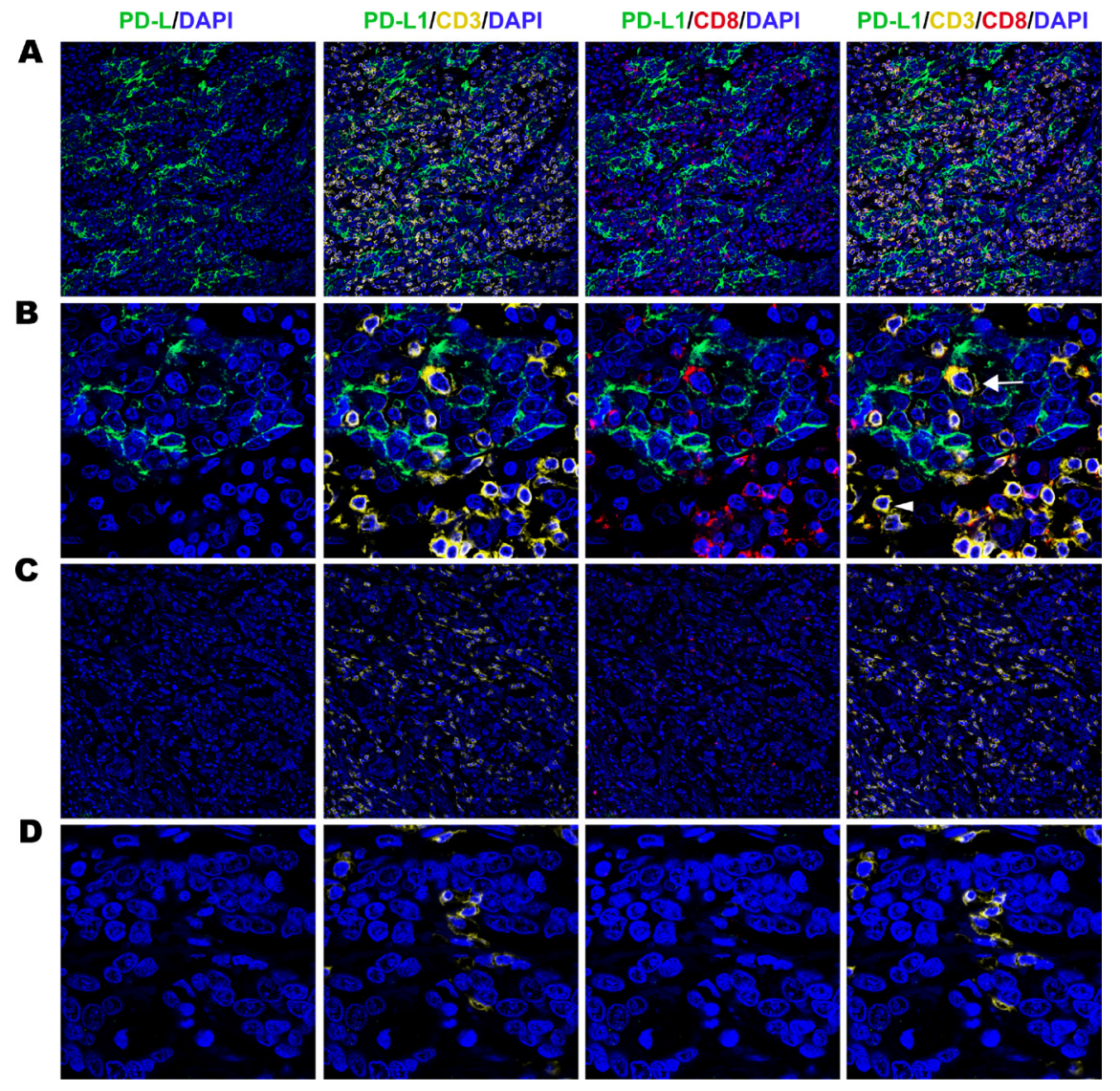

Figure 2: Fluorescent multiplex immunohistochemistry (mIHC) staining pattern for tumour cell PD-L1 and TILs in gastric adenocarcinoma tissues. (A) Strong expression of PD-L1 on tumour cells with high density of tumour infiltrating CD3+ and CD8+ cells (original magnification $\times 100$ ). (B) Strong expression of PD-L1 on tumour cells with high density of tumour infiltrating $\mathrm{CD} 3+$ cells (white arrow) and CD3 $+\mathrm{CD} 8+$ cells (white arrowhead) (original magnification $\times 400)$. (C) Negative expression PD-L1 on tumour cells with low density of tumour infiltrating CD3+ and CD8+ cells (original magnification $\times 100$ ). (D) Negative expression PD-L1 on tumour cells with low density of tumour infiltrating CD3+ and CD8+ cells (original magnification $\times 400$ ). 
cells (Figure 2C). Especially the CD3+CD8+ cells was much fewer in PD-L1 negative subset (Figure 2D). In the PD-L1 positive immune cell subset similar results were observed. PD-L1 negative immune cell cases were infiltrated with low density of CD3+ TILs and CD8+ TILs (Figure 3A). High density of CD3+ TILs and CD8+ TILs were observed in PD-L1 positive immune cell cases (Figure 3B). Approximately 80\% PD-L1+ immune cells were PD-L1+ CD3+ and $60 \%$ were PD-L1+CD3+CD8+ (Figure 3C).

Tumor infiltrating CD3+ and CD8+ cells were counted, scored as $1(<1 \%), 2(1-9 \%), 3(10-20 \%)$, or $4(>20 \%)$ and classified into low and high subsets. The correlation between density of different TIL types and PDL1 expression was summarized in Table 2. Approximately $47 \%$ had a high density of CD3 + TILs and 46\% had a high density of CD8+ TILs (Table 2). In the PD-L1 positive tumour cell subset, a significantly higher proportion of CD3+ TILs and CD8+ TILs were observed. In the PDL1 positive tumour cell subset, approximately $62 \%$ had a high density of CD3+ TILs and CD8+ TILs. In contrast, only $38 \%$ of the PD-L1 negative immune cell subset had a high density of CD3 + TILs, 37\% had a high density of CD8+ TILs. In the PD-L1 positive immune cell cases, approximately $66 \%$ had a high density of CD3+ TIL and
CD8+ TILs. In contrast, only $29 \%$ of the PD-L1 negative immune cell subset had a high density of CD3+ TILs, 27\% had a high density of CD8+ TILs.

These results demonstrated that PD-L1 expression in tumor cells and immune cells was positively associated with the density of CD3+ and CD8+ TILs.

\section{Survival analysis based on PD-L1 expression and TILs}

We performed a Kaplan-Meier analysis and Log-Rank test to assess the prognostic role of PD-L1 expression in gastric cancer. PD-L1 expression in tumour cells was associated with worse prognosis. The PD-L1 positive tumour cell subset had a 14.2 month cumulative survival and the PD-L1 negative tumour cell subset had an 18.6 month cumulative survival time (Figure 4A). The difference in cumulative survival between PD-L1 positive and PD-L1 negative subsets in the whole population was significant $(P=0.025)$. There was no significant difference $(P=0.138)$ in cumulative survival time between PD-L1 positive and PD-L1 negative immune cell subsets in the whole population (Figure 4B). Previous reports indicated that the density of TILs was associated with prognosis of cancer patients [10, 16-19]. Significant longer cumulative
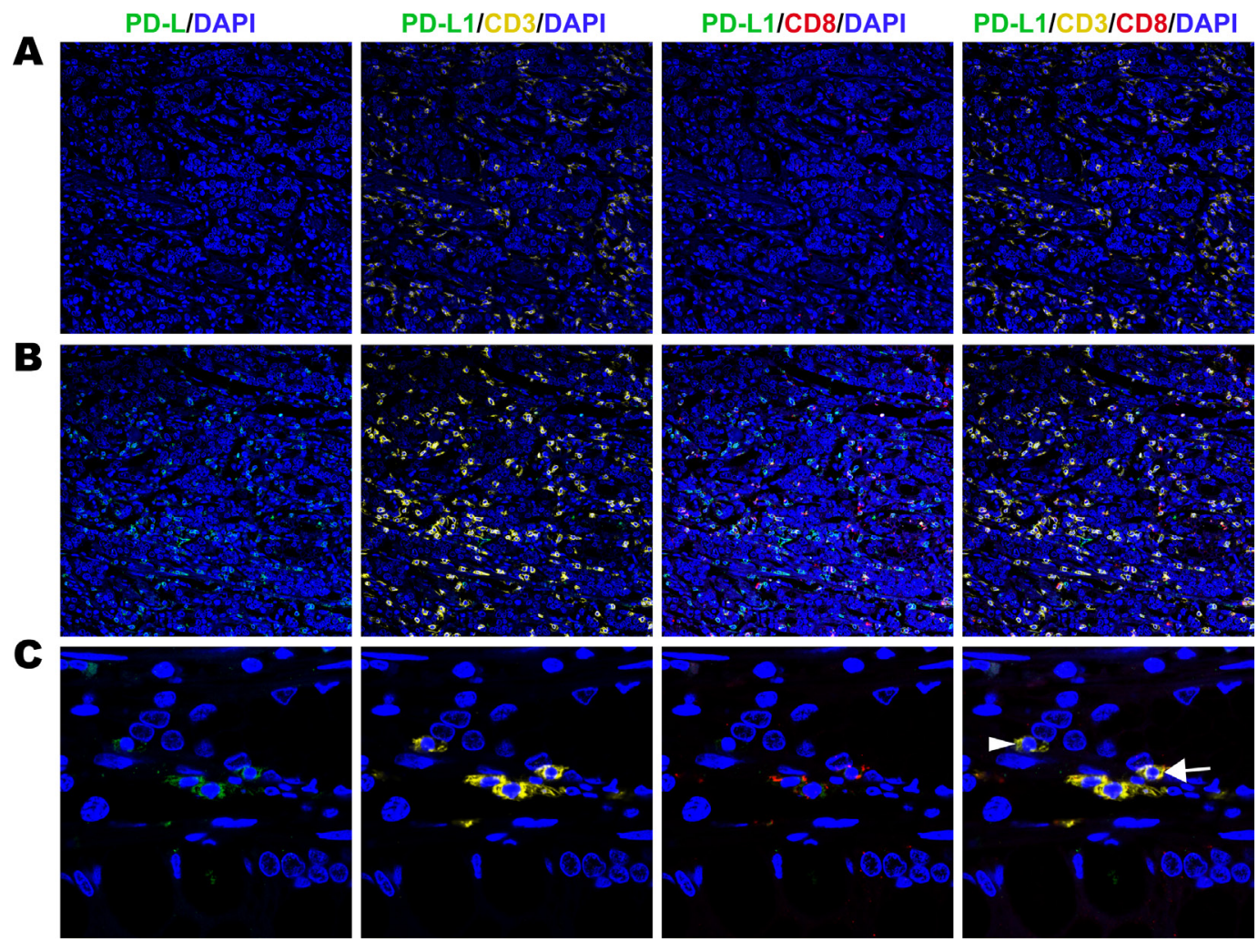

Figure 3: Fluorescent multiplex immunohistochemistry (mIHC) staining pattern for immune cell PD-L1 and TILs in gastric adenocarcinoma tissues. (A) Negative expression of PD-L1 on immnune cells with low density of tumour infiltrating CD3+ and CD8+ cells (original magnification $\times 100$ ). (B) Positive expression of PD-L1 on immune cells with high density of tumour infiltrating CD3+ cells and CD8+ cells (original magnification $\times 100)$. (C) Positive expression PD-L1 on tumour cells with low density of tumour infiltrating PD-L1+CD3+ (white arrow) and PD-L1+CD3+CD8+ cells (white arrowhead) (original magnification $\times 400$ ). 
Table 2: Relationship of PD-L1 expression and T cell density in gastric cancer

\begin{tabular}{|c|c|c|c|c|c|c|}
\hline \multirow{2}{*}{ Characteristic } & \multicolumn{2}{|c|}{ CD3 } & \multirow{2}{*}{$P$-Value } & \multicolumn{2}{|c|}{ CD8 } & \multirow{2}{*}{$P$-Value } \\
\hline & Low & High & & Low & High & \\
\hline Total no & $56(53 \%)$ & $49(47 \%)$ & & $57(54 \%)$ & $48(46 \%)$ & \\
\hline Tumor cells & & & 0.025 & & & 0.015 \\
\hline PD-L1 Negative & $42(62 \%)$ & $26(38 \%)$ & & $43(63 \%)$ & $25(37 \%)$ & \\
\hline PD-L1 Positive & $14(48 \%)$ & $23(62 \%)$ & & $14(48 \%)$ & $23(62 \%)$ & \\
\hline Immune cells & & & 0.001 & & & 0.000 \\
\hline PD-L1 Negative & $39(71 \%)$ & $16(29 \%)$ & & $40(73 \%)$ & $15(27 \%)$ & \\
\hline PD-L1Positive & $17(34 \%)$ & $33(66 \%)$ & & $17(34 \%)$ & $33(66 \%)$ & \\
\hline
\end{tabular}

survival time was observed in high density CD3+ and CD8+ subsets (Figure 4C, 4D).

We found PD-L1 expression was association with density of CD3+ and CD8+ TILs. PD-L1 expression was combined with TILs density and cumulative survive was analysed. Patients were classified into four groups depend on the PD-L1 expression and density of TILs (Figure 5). We observed the longest cumulative survival time in $\mathrm{T}$
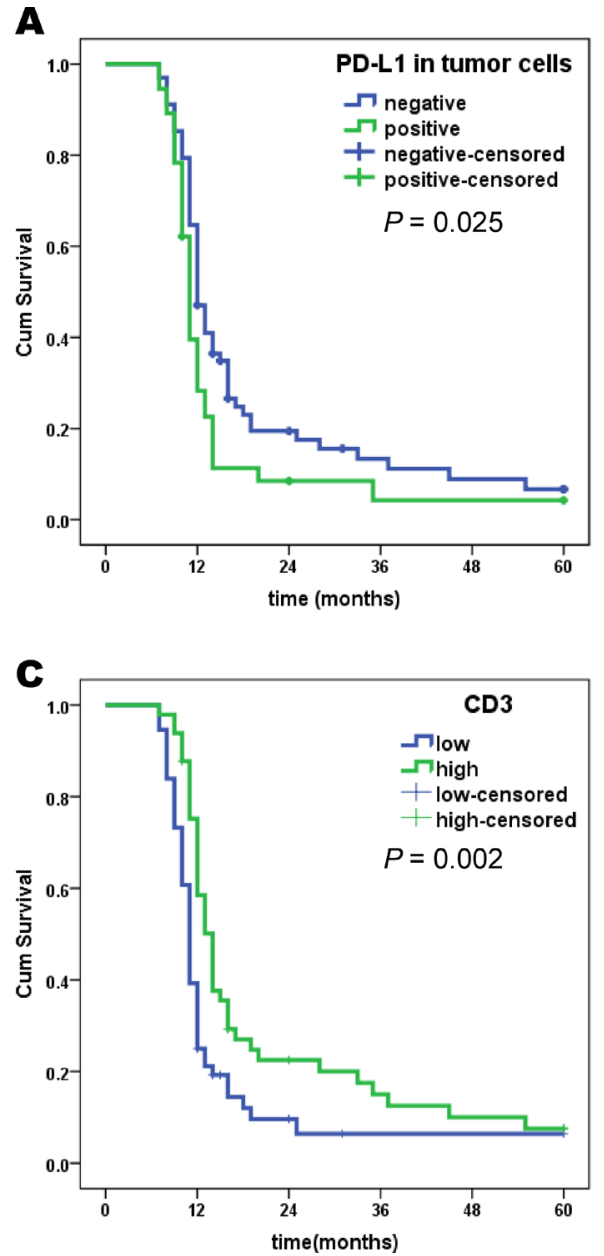

PD-L1-/TILs high group. The shortest survival time was observed in T PD-L1+/TILs low group (Figure 5A, 5B). Difference of survival times were significantly. Similarly, PD-L1 expression in immune cells were classified into four groups. The Im PD-L1+/CD3+ low group had the shortest survival time (Figure 5C). However, no significant difference was observed. The Im PD-L1+/CD $8+$ high group had a significant longer cumulative survival than
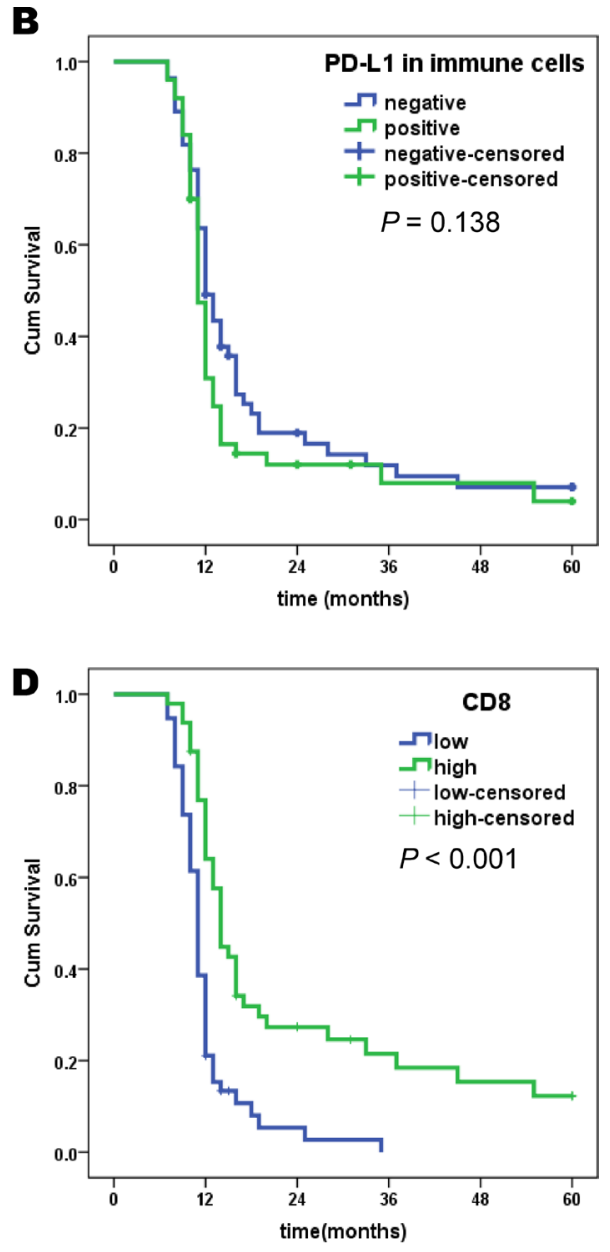

Figure 4: Kaplan-Meier survival analysis with Log-Rank test PD-L1 and TILs. (A) Survival curves of PD-L1 positive and negative in tumour cells. (B) Survival curves of PD-L1 positive and negative in immune cells. (C) Survival curves of low-density and high-density CD3+ TILs. (D) Survival curves of low-density and high-density CD8+ TILs. 
Im PD-L1+/ CD8+ low group (Figure 5D). Interestingly, Im PD-L1+/CD8+ high group had a longer cumulative survival than Im PD-L1-/CD8+ low group (Figure 5D). However, no significant difference was observed. These results indicated that combination of PD-L1 with pre-excited TILs may be more precise than PD-L1 alone for predicting survival in gastric cancer.

\section{DISCUSSION}

PD-L1 expression on tumour cells has been correlated with tumour cell evasion through down regulation of active $\mathrm{T}$ cell mediated immune responses [20, 21]. PDL1 expression is found in approximately $12 \%$ to $46 \%$ of gastric cancer patients, suggesting this may be a predictive biomarker for successful PD1/PD-L1 immune check point inhibitor therapy. Expression of PD-L1 has also been shown to be an independent prognostic predictor in gastric cancer.

In this study, we demonstrated that PD-L1 was expressed on tumour cell in $35 \%$ of gastric cancer cases. In $48 \%$ of gastric cancer cases, PD-L1 was expressed on immune cells. This result was consistent with previous reports. However, even in the PD-L1 positive sample, the overall proportion of PD-L1 stained tumour cells and immune cells was low. We analysed the correlation between PD-L1 expression and several clinicopathologic parameters in gastric cancer. PD-L1 positive cases were found more frequently in tumours located in gastric antrum and in poorly differentiated tumours. No significant associations were found for other parameters, including age, disease stage, and lymph node metastasis. Interestingly, PD-L1 expression in tumour cells correlated significantly with the Ki67 and HER-2 status of gastric cancer. Our data indicated that PD-L1 expression in tumour cells associated with poor-prognostic features such as poor differentiation and high proliferation. This result suggests future investigations of these molecules could be conducive to anti-tumour immunotherapy for gastric cancer.

It has been shown that PD-L1 expression was associated with overall survival in gastric cancer and weak tumour staining of PD-L1 was associated with better overall survival time compared to strong tumour staining of PD-L1 [15-17]. In the present study, we sorted cases into positive and negative subsets according to the percentage of PD-L1 staining cells. We observed that PDL1 expression on tumour cells was associates with poorprognostic features, but we did not find a significantly

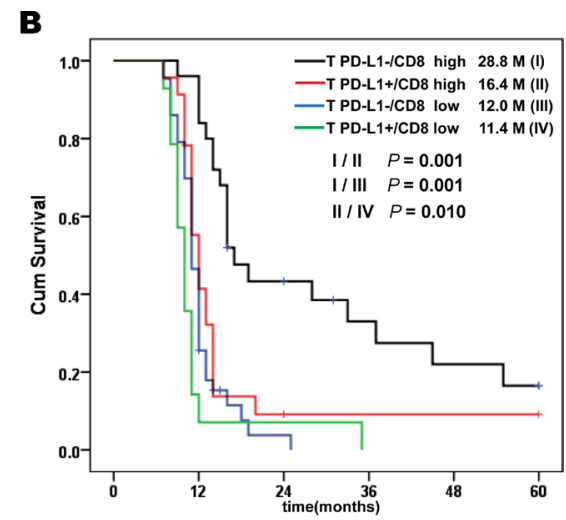

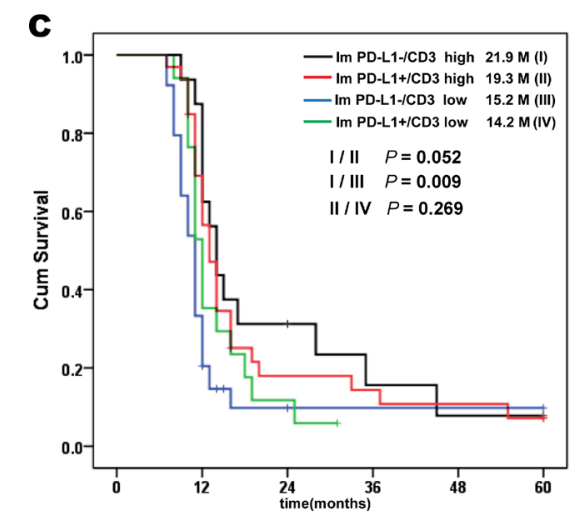

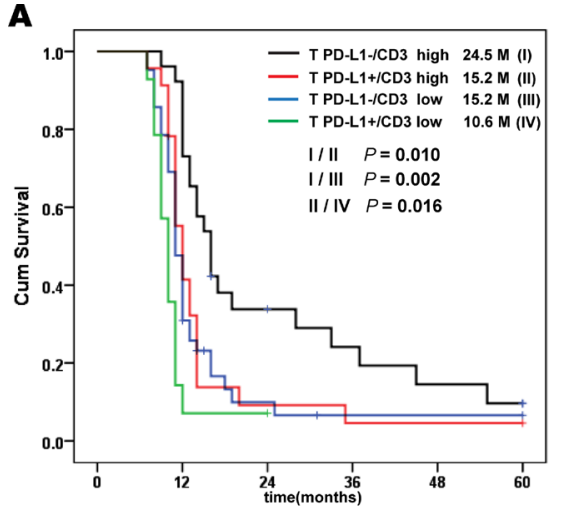

D

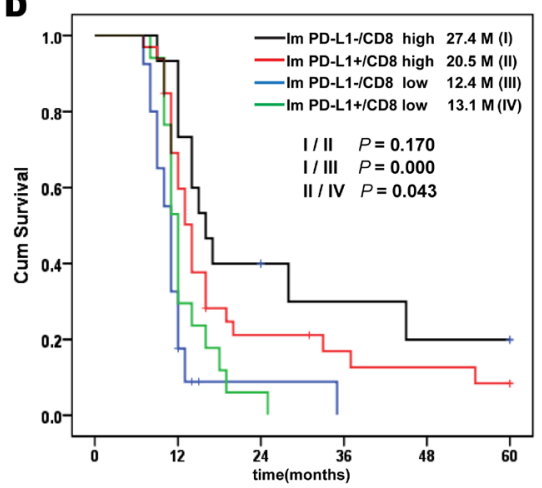

Figure 5: Kaplan-Meier survival analysis with Log-Rank test of PD-L1 combined with TILs. (A) Survival curves of positive and negative expression of PD-L1 on tumour cells with low density and high density tumour infiltrating CD3+ cells. (B) Survival curves of positive and negative expression of PD-L1 on tumour cells with low density and high density tumour infiltrating CD8+ cells. (C) Survival curves of positive and negative expression of PD-L1 on immune cells with low density and high density tumour infiltrating CD3 + cells. (D) Survival curves of positive and negative expression of PD-L1 on immune cells with low density and high density tumour infiltrating CD $8+$ cells. 
different survival time with or without PD-L1 expression on immune cells. It indicated that PD-L1 expression in tumour cells possibly be an independent predictor biomarkes but not in immune cells.

Previous studies demonstrated that PD-L1 expression strongly correlated with TILs [22]. The density of CD8+ TILs are associated with PD-L1 expression in many type of cancers, such as melanoma [23], human brain metastases, lung cancer [24] and gastric cancer [16]. In colorectal cancer tissue, tumour CD247 (PDL1) is inversely associated with FoxP3+, but not CD3+, CD8+ or CD45RO+ cell density [25]. Our current study observed PD-L1 expression in tumour cells and immune cells was strongly correlated a high density of CD3+ and CD8+ TILs. And more than 70\% CD3+ cells were CD3+ CD8+ double positive cells. Approximately $80 \%$ PD-L1+ immune cells were PD-L1+ CD3+ and $60 \%$ were PD-L1+ CD3+CD8+ cells. Significantly different survival times were observed between groups of T PD-L1+/TILs high, T PD-L1-/TILs high, T PD-L1+/TILs low and T PD-L1-/ TILs low. The T PD-L1+/CD8+ low group had the shortest survival. Accordingly, T PD-L1-/CD8+ high group had the best prognosis. Similar the Im PD-L1+/CD8+ high group had a better outcome than Im PD-L1+/CD8+ low group. Interestingly, Im PD-L1+/CD8+ low group had a longer survival time than PD-L1-/CD8+ low group. Although no significant difference was observed. It suggested that PD-L1 expression in immune cells combined with TILs may have different prognostic value. Larger samples and improved methods may be required for the further studies.

In conclusion, PD-L1 expression on tumour cells and immune cells was positively associated with densities of CD3+ and CD8+ TILs. Up-regulated PD-L1 expression with low density TILs indicated the worst prognosis for gastric cancer patients. Up-regulated PD-L1 expression on tumor cells and immune cells with increased density of CD8+ cells was indicated an improved prognosis than low density of CD8+ TILs. Combination of PD-L1 with preexcited TILs may be more important than PD-L1 alone for predicting survival in gastric cancer. These findings may have important implications for PD-1/PD-L1 block therapy in gastric cancer patients.

\section{MATERIALS AND METHODS}

\section{Patients and samples}

We collected gastric cancer specimens from 105 patients who were diagnosed and undergoing surgical excision of a primary tumour at the First People's Hospital of Zhenjiang between January 2009 and June 2011. The characteristics and pathological data of the patients were collected from the pathology department of the First People's Hospital of Zhenjiang. We excluded patients who received radiotherapy, chemotherapy or other medical intervention before the study. All of the study subjects had completed the follow-up process until July 2016. All the tissue specimens were fixed in formalin and embedded in paraffin for IHC analysis.

\section{Immunohistochemical analysis}

Formalin-fixed and paraffin-embedded samples were obtained from the pathology department of the First People's Hospital of Zhenjiang. Approximately 4- $\mu \mathrm{m}$ thick sections were cut from the selected samples. The sections were mounted on glass slides and then used for $\mathrm{H} \& \mathrm{E}$, IHC and $\mathrm{mIHC}$ staining.

The diagnosis was verified for all samples using H\&E staining. For IHC, sections were deparaffinised, rehydrated and incubated in Antigen retrieval Citra solution, pH 6.0 (BOSTER, Wuhan, China) at $95^{\circ} \mathrm{C}-99^{\circ} \mathrm{C}$ for $20 \mathrm{~min}$, then cooled to room temperature. Slides were incubated in $3 \% \mathrm{H}_{2} \mathrm{O}_{2}$ (BOSTER, Wuhan, China) for 10 min at room temperature to block endogenous enzyme. Then, 5\% BSA (BOSTER, Wuhan, China) was used to block unspecific binding for $20 \mathrm{~min}$ at room temperature. For IHC, sections were incubated with 1:250 diluted primary antibody PD-L1 (clone: E1L3N, Cell Signaling Technology, MA USA.MA USA) incubated at $4^{\circ} \mathrm{C}$ overnight. Anti- Rabbit IgG SABC kit (SA1020, BOSTER, Wuhan, China) and DAB detection kit (ZSGB-BIO, Beijing, China) were used. For Fluorescent Multiplex immunohistochemistry (mIHC), firstly, slides were incubated in 1:250 diluted primary antibody CD8 (clone: C8/144B, PD-L1/CD3\&/ CD8 $\alpha$ /Multiplex IHC Antibody Panel \#65713, Cell Signaling Technology, MA USA.) for 60 minutes at room temperature. After wash, slides were incubated in HRP labeled anti-mouse secondary antibody (SV0001, BOSTER, Wuhan, China) for 30 minutes at room temperature. Wash slides by TNT buffer (0.1 M Tris-HCl, pH 7.5, 0.15M NaCl, 0.05\% Tween 20). Sections were keep in dark place and incubated with 1:50 diluted TSA Plus Cyanine 5 (TSA Plus Fluorescence NEL745001KT, PerkineElmer, MA USA) for 5 minutes at room temperature. Slides were stripped in $10 \mathrm{mM}$ sodium citrate buffer, $\mathrm{pH} 6.0$ at a sub-boiling temperature for 10 min by microwave. Secondary, 1:250 diluted primary antibody CD3 (clone:D7A6E, PD-L1/CD3e/CD8 $\alpha$ / Multiplex IHC Antibody Panel \#65713, Cell Signaling Technology, MA USA.) were add on the sections and incubated in dark for 60 minutes at room temperature. HRP labeled anti-rabbit secondary antibody (SV0002, BOSTER, Wuhan, China) and 1:50 diluted TSA Plus Cyanine 3 (TSA Plus Fluorescence NEL744001KT, PerkineElmer, MA USA) were used. After stripping, slides were incubated with 1:250 diluted PD-L1 (clone: E1L3N, PD-L1/CD3ع/ CD8 $\alpha$ /Multiplex IHC Antibody Panel \#65713, Cell Signaling Technology, MA USA.) for 60 minutes at room temperature. HRP labeled anti- 
rabbit secondary antibody (SV0002, BOSTER, Wuhan, China) and 1:50 diluted TSA Plus Fluorescein (TSA Plus Fluorescence NEL741001KT, PerkineElmer, MA USA) were used. Finally, mount sections with coverslips using DAPI (P36941, ProLong ${ }^{\circledR}$ Gold Antifade Mountant with DAPI, Thermo Fisher). Leica Laser confocal microscopy was used for Multi-Color Fluorescence Imaging.

All IHC and mIHC analyses were performed by two independent observers (Shen and Huang) who were unaware of the patients' record of clinicopathological features. PD-L1 expression in tumour cells was graded into three groups: 0 (no staining), 1 (weak staining), 2 (moderate to intense staining). We counted PD-L1 staining cells per 1000 tumor cells. The percentage of PD-L1 expression tumuor cells was determined based on 10 random areas (HPF $400 \times$ magnification) in each section and the values were average for statistical analysis. Cases in group 1 or 2 with $\geq 5 \%$ PD-L1 in tumour cells were considered positive as previously. Immune cells were scored separately. PD-L1 expression immune cells (intratumoral) were counted per 1000 immune cells, and the percentage was determined based on 10 random areas (HPF $400 \times$ magnification) in each section and the values were average for statistical analysis. Cases with $\geq 1 \%$ PD-L1 in immune cells were considered positive. The number and percentage of CD3 and CD8 expression TILs were determined as PDL1. Expression of CD3 and CD8 in TILs was scored as $1(<1 \%), 2(1-9 \%), 3(10-20 \%)$, or $4(>20 \%)$. Subsequently, the grades were classified into low (scores 1-2) and high (scores 3-4) subsets. The IHC results for Ki67 and HER-2 expression were obtained from the pathology department of the First People's Hospital of Zhenjiang.

\section{Statistical analyses}

All statistical analyses were performed using SPSS 22.0 (IBM Corporation, New York, USA). Pearson's chi-square test and Fisher's exact test were performed to evaluate the correlation between PD-L1 expression, density of TILs and other clinicopathological features. Kaplan-Meier methods were used to calculate cumulative survival and 95\% confidence intervals (CI) were computed. Log-Rank test was used to determine significant difference between survival curves. Student $t$-test was used to analyze the significance of differences between the mean values of two variables. All comparisons were calculated using a two-tailed test. Significant differences were considered at $P<0.05$.

\section{CONFLICTS OF INTEREST}

The authors declare that they have no conflicts of interest.

\section{FUNDING}

This work was supported by the National Natural Science Foundation of China (81502088, 81502621 and 81300287), the Start-Up Research Funding of Jiangsu University for Distinguished Scholars (15JDG021) and China Postdoctoral Science special Foundation (2017M5654).

\section{REFERENCES}

1. Torre LA, Bray F, Siegel RL, Ferlay J, Lortet-Tieulent J, Jemal A. Global Cancer Statistics, 2012. CA Cancer J Clin. 2015; 65:87-108.

2. Jemal A, Bray F, Center MM, Ferlay J, Ward E, Forman D. Global Cancer Statistics. CA Cancer J Clin. 2011; 61:69-90.

3. Topalian SL, Hodi FS, Brahmer JR, Gettinger SN, Smith DC, McDermott DF, Powderly JD, Carvajal RD, Sosman JA, Atkins MB, Leming PD, Spigel DR, Antonia SJ, et al. Safety, activity, and immune correlates of anti-PD-1 antibody in cancer. N Engl J Med. 2012; 366:2443-54.

4. Brahmer JR, Tykodi SS, Chow LQ, Hwu WJ, Topalian SL, Hwu P, Drake CG, Camacho LH, Kauh J, Odunsi K, Pitot HC, Hamid O, Bhatia S, et al. Safety and activity of antiPD-L1 antibody in patients with advanced cancer. N Engl J Med. 2012; 366:2455-65.

5. Wolchok JD, Kluger H, Callahan MK, Postow MA, Rizvi NA, Lesokhin AM, Segal NH, Ariyan CE, Gordon RA, Reed K, Burke MM, Caldwell A, Kronenberg SA, et al. Nivolumab plus ipilimumab in advanced melanoma. N Engl J Med. 2013; 369:122-33.

6. Robert C, Long GV, Brady B, Dutriaux C, Maio M, Mortier L, Hassel JC, Rutkowski P, McNeil C, Kalinka-Warzocha E, Savage KJ, Hernberg MM, Lebbé C, et al. Nivolumab in previously untreated melanoma without BRAF mutation. N Engl J Med. 2015; 372:320-30.

7. Muro K, Chung HC, Shankaran V, Geva R, Catenacci D, Gupta S, Eder JP, Golan T, Le DT, Burtness B, McRee AJ, Lin CC, Pathiraja K, et al. Pembrolizumab for patients with PD-L1-positive advanced gastric cancer (KEYNOTE-012): a multicentre, open-label, phase $1 \mathrm{~b}$ trial. Lancet Oncol. 2016; 17:717-26.

8. Freeman GJ, Long AJ, Iwai Y, Bourque K, Chernova T, Nishimura H, Fitz LJ, Malenkovich N, Okazaki T, Byrne MC, Horton HF, Fouser L, Carter L, et al. Engagement of the PD-1 immunoinhibitory receptor by a novel B7 family member leads to negative regulation of lymphocyte activation. J Exp Med. 2000; 192:1027-34.

9. Dong H, Strome SE, Salomao DR, Tamura H, Hirano F, Flies DB, Roche PC, Lu J, Zhu G. Tumor-associated B7-H1 promotes T-cell apoptosis: a potential mechanism of immune evasion. Nat Med. 2002; 8:793-800.

10. Marincola FM, Jaffee EM, Hicklin DJ, Ferrone S. Escape of human solid tumors from T-cell recognition: molecular 
mechanisms and functional signi cance. Adv Immunol. 2000; 74:181-273.

11. Iwai Y, Ishida M, Tanaka Y, Okazaki T, Honjo T, Minato N. Involvement of PD-L1 on tumor cells in the escape from host immune system and tumor immunotherapy by PD-L1 blockade. Proc Natl Acad Sci U S A. 2002; 99:12293-7.

12. Kim JW, Nam KH, Ahn SH, Park DJ, Kim HH, Kim SH, Chang H, Lee JO, Kim YJ, Lee HS, Kim JH, Bang SM, Lee JS, et al. Prognostic implications of immunosuppressive protein expression in tumors as well as immune cell in 1tration within the tumor microenvironment in gastric cancer. Gastric cancer. 2016; 19:42-52.

13. Böger C, Behrens HM, Mathiak M, Krüger S, Kalthoff $H$, Röcken C. PD-L1 is an independent prognostic predictor in gastric cancer of Western patients. Oncotarget. 2016; 7:24269-83. https://doi.org/10.18632/oncotarget.8169.

14. Sabatier R, Finetti P, Mamessier E, Adelaide J, Chaffanet M, Ali HR, Viens P, Caldas C, Birnbaum D, Bertucci F. Prognostic and predictive value of PDL1 expression in breast cancer. Oncotarget. 2015; 6:5449-64. https://doi. org/10.18632/oncotarget.3216.

15. Tamura T, Ohira M, Tanaka H, Muguruma K, Toyokawa T, Kubo N, Sakurai K, Amano R, Kimura K, Shibutani M, Maeda K, Hirakawa K. Programmed Death-1 Ligand-1 (PDL1) Expression Is Associated with the Prognosis of Patients with Stage II/III Gastric Cancer. Anticancer Res. 2015; 5:5369-76.

16. Thompson ED, Zahurak M, Murphy A, Cornish T, Cuka N, Abdelfatah E, Yang S, Duncan M, Ahuja N, Taube JM, Anders RA, Kelly RJ. Patterns of PD-L1 expression and CD8 $\mathrm{T}$ cell in ltration in gastric adenocarcinomas and associated immune stroma. Gut. 2017; 66:794-801.

17. Zhang L, Qiu M, Jin Y, Ji J, Li B, Wang X, Yan S, $\mathrm{Xu}$ R, Yang D. Programmed cell death ligand 1 (PD-L1) expression on gastric cancer and its relationship with clinicopathologic factors. Int J Clin Exp Pathol. 2015; 8:11084-91.
18. Hou J, Yu Z, Xiang R, Li C, Wang L, Chen S, Li Q, Chen M, Wang L. Correlation between in 1tration of FOXP3+ regulatory $\mathrm{T}$ cells and expression of $\mathrm{B} 7-\mathrm{H} 1$ in the tumor tissues of gastric cancer. Exp Mol Pathol. 2014; 96:284-91.

19. Kim KJ, Lee KS, Cho HJ, Kim YH, Yang HK, Kim WH, Kang GH. Prognostic implications of tumor-in ltrating FoxP3+ regulatory $\mathrm{T}$ cells and $\mathrm{CD} 8+$ cytotoxic $\mathrm{T}$ cells in microsatellite-unstable gastric cancers. Hum Pathol. 2014; 45:285-93.

20. Ostrand-Rosenberg S, Horn LA, Haile ST. The programmed death-1 immune-suppressive pathway: barrier to antitumor immunity. J Immunol. 2014; 193:3835-41.

21. Rooney MS, Shukla SA, Wu CJ, Getz G, Hacohen N. Molecular and Genetic Properties of Tumors Associated with Local Immune Cytolytic Activity. Cell. 2015; 160:48-61

22. Harter PN, Bernatz S, Scholz A, Zeiner PS, Zinke J, Kiyose M, Blasel S, Beschorner R, Senft C, Bender B, Ronellenfitsch MW, Wikman $H$, Glatzel $M$, et al. Distribution and prognostic relevance of tumor-infiltrating lymphocytes (TILs) and PD-1/PD-L1 immune checkpoints in human brain metastases. Oncotarget. 2015; 6:40836-49. https://doi.org/10.18632/oncotarget.5696.

23. Tumeh PC, Harview CL, Yearley JH, Shintaku IP, Taylor EJ, Robert L, Chmielowski B, Spasic M, Henry G, Ciobanu V, West AN, Carmona M, Kivork C, et al. PD-1 blockade induces responses by inhibiting adaptive immune resistance. Nature. 2014; 515:568-71.

24. Kim MY, Koh J, Kim S, Go H, Jeon YK, Chung DH. Clinicopathological analysis of PD-L1 and PD-L2 expression in pulmonary squamous cell carcinoma: Comparison with tumor-in ltrating $\mathrm{T}$ cells and the status of oncogenic drivers. Lung cancer. 2015; 88:24-33.

25. Masugi Y, Nishihara R, Yang J, Mima K, da Silva A, Shi Y, Inamura K, Cao Y, Song M, Nowak JA, Liao X, Nosho K, Chan AT, et al. Tumour CD274 (PD- L1) expression and T cells in colorectal cancer. Gut. 2017; 66:1463-1473. 